\begin{tabular}{|l|l|l|l|l|}
\hline Cuadernos de Investigación Geográfica & 2003 & N $^{\circ} 29$ & pp. 7-21 & ISSN 0211-6820 \\
\hline
\end{tabular}

(C) Universidad de La Rioja

\title{
INFLUENCIA DE LOS EMBALSES SOBRE EL RÉGIMEN FLUVIAL EN LOS PIRINEOS CENTRALES
}

\author{
J.I. LÓPEZ MORENO(*) \\ J.M. GARCÍA RUIZ \\ Instituto Pirenaico de Ecología, CSIC, Campus de Aula Dei, Apdo. 202, 50080- Zaragoza
}

(*)nlopez@ipe.csic.es

\begin{abstract}
RESUMEN: Se estudia el cambio de régimen fluvial aguas abajo de los embalses o sistemas de embalses situados en los ríos del Pirineo Central, entre las cuencas de los ríos Aragón y Noguera Pallaresa. La gestión de los embalses pretende almacenar agua durante una parte del año para liberar caudales más elevados durante los meses que lo requiere la demanda (agrícola, producción hidroeléctrica). Se ha observado que los cambios en el régimen fluvial son muy variables tanto en intensidad como en la estacionalidad en que los caudales son redistribuidos. El ratio de retención de cada embalse o sistema de embalses, el uso que desempeñan y la ubicación de cada embalse dentro del sistema en el que están integrados, explican la mayor parte de la varianza del cambio observado en la zona de estudio.

ABSTRACT: Changes on river flow regimes downstream of reservoirs or multireservoir systems located in the Central Pyrenees (between Aragón river and Noguera Pallaresa river) is studied. The reservoir management aims to store water during a part of the year in order to release relatively high flows for agricultural or hydropower purposes. A high variability in the intensity of the fluvial regime change and in the seasonality of the released discharges has been observed. The impounded ratio index, the aim of the reservoir and the location of the reservoir into the multireservoir system explain most of the observed variance in the study area.
\end{abstract}

Palabras clave: Cambio de régimen fluvial, ratio de retención, gestión de embalses, Pirineo Central.

Key words: Change on river flows, impounded ratio index, reservoir management, Central Pyrenees.

\section{Introducción}

Los embalses son una herramienta fundamental en la gestión de los recursos hídricos. La construcción de grandes embalses se ha incrementado mucho en las últimas décadas. En el siglo XX el número de grandes embalses (en los que la presa supera los $15 \mathrm{~m}$ de altura) ha pasado en todo el mundo de 500 a más de 42.000 (Morris \& Fan, 1997) y la capacidad de embalsado se estima alrededor de $6.000 \mathrm{Km}^{3}$, lo que representa el $13 \%$ de la escorrentía. En 
1994 más de 1200 presas estaban en construcción, de las que el 6,4\% superaban los 100 m de altura (Takeuchi, 1998).

La regulación de los caudales permite satisfacer diversas demandas, como proveer de agua a ciudades e industrias, producción hidroeléctrica y suministro a zonas de regadío a través de canales a los que se deriva parte del agua embalsada. En muchas ocasiones los embalses son gestionados para hacer frente a eventos hidrológicos extremos, reduciendo la intensidad de las avenidas (López Moreno et al. 2002), o manteniendo un caudal ecológico durante periodos de estiaje.

Durante la segunda mitad del siglo XX se han construido numerosos embalses en los Pirineos debido: i) al incremento de la producción hidroeléctrica en ríos dotados de elevados caudales e importantes desniveles en su pérfil longitudinal; ii) la necesidad de suministro de agua para nuevas zonas de regadío (Bardenas, Sistema de Riegos del Alto Aragón-Monegros) y asentamientos urbanos. Además, los contrastes litológicos existentes en el Pirineo han generado morfologías que han facilitado la construcción de presas. De esta forma, la totalidad de los principales ríos pirenaicos se encuentran regulados por embalses o sistemas de embalses, capaces de almacenar buena parte de la aportación anual que reciben, especialmente en los ríos Cinca y Noguera Ribagorzana.

Los embalses causan importantes cambios hidrológicos y geomorfológicos aguas abajo de la presa (Al Taieee et al., 1990; Brandt, 2000; Higgs y Petts, 1998; Sammut et al. 1995). Las principales afecciones que se pueden observar son: i) cambios en la calidad del agua a la salida del embalse, siendo la disminución de la carga de fondo y del sedimento en suspensión y la variación en la temperatura del agua los más frecuentes (Andrews, 1986); ii) reducción de caudales debido a la derivación de parte de la aportación a través de canales (Adams, 1985); iii) reducción del número e intensidad de crecidas, frecuentemente laminadas con el fin de incrementar el volumen embalsado y reducir posibles daños aguas abajo de la presa (Pircher, 1990; Zsuffa, 1999; López et al., 2002) y iv) cambios en el régimen fluvial (Bonacci y Roje-Bonacci, 2003).

En este trabajo se analizan los cambios de los regímenes de los ríos pirenaicos aguas abajo de los embalses o sistemas de embalses, así como los factores que explican la distinta intensidad del cambio de régimen fluvial. La estacionalidad de los caudales refleja las características climáticas y fisiográficas de las cuencas drenadas, pero la gestión de los embalses puede modificar de una forma muy notable la distribución anual de los caudales. Cambios en el régimen fluvial pueden tener un impacto en los ecosistemas (Baxter, 1977; Giers et al., 1998), y en la disponibilidad de recursos hídricos de los sectores situados aguas abajo de las presas.

\section{2. Área de estudio}

El área de estudio, entre la cuenca alta del río Aragón y la del río Noguera Pallaresa, ocupa una parte importante del Pirineo Central español (Fig. 1). Las máximas altitudes se encuentran en las cabeceras de los ríos Gállego, Cinca, Ésera y Noguera Ribagorzana, donde se superan ocasionalmente los $3000 \mathrm{~m}$ de altitud. 


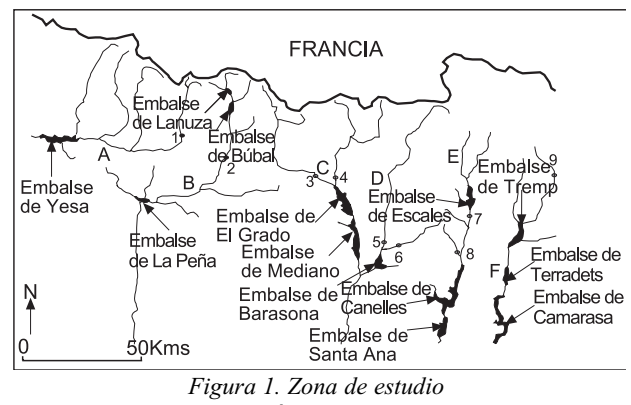

A: Río Aragón; B: Río Gállego; C: Río Cinca; D: Río Ésera; E: Río Noguera Ribagorzana; F: Río Noguera Pallaresa

1: Jaca; 2: Sabiñánigo; 3: Boltaña; 4: Ainsa; 5: Graus; 6: Capdella; 7 Pont de Suert; 8: Puente de Montañana; 9: Sort

Desde un punto de vista estructural, el relieve se organiza en bandas paralelas de norte a sur, distinguiéndose: i) el Pirineo Axil, formado por materiales paleozoicos que muestran un comportamiento muy heterogéneo ante la erosión; ii) las Sierras Interiores, con materiales mesozoicos (básicamente calizas y arenicas), dan lugar a un relieve muy abrupto; iii) el Sector del Flysch, formado por areniscas y margas del Eoceno, alternando en estratos muy delgados e intensamente plegados; iv) la Depresión Interior Altoaragonesa modelada sobre margas del Eoceno, su altitud se sitúa entre 600 y 900 m; v) las molasas prepirenaicas, al sur de las margas eocenas, están formadas por areniscas, arcillas y margas, con potentes acumulaciones de conglomerados en su borde septentrional; y finalmente, vi) las Sierras Exteriores, formadas por margas, calizas, areniscas y arcillas yesíferas, dan lugar a una alineación estrecha y continua que recorre el sector meridional de la zona de estudio.

La mayor parte de los embalses estudiados aprovechan (i) la existencia de cubetas de sobreexcavación glaciar en los sectores más elevados de la zona de estudio (cabecera del río Gállego), y (ii) amplias cubetas erosivas desarrolladas a partir de litologías más blandas, cerradas frecuentemente en el contacto con materiales más resistentes. Los de mayores dimensiones ocupan estas últimas ubicaciones.

Las precipitaciones se distribuyen siguiendo un doble gradiente norte-sur y oesteeste, de acuerdo con la mayor o menor influencia atlántica y mediterránea y con los grandes rasgos del relieve. Un ambiente oceánico domina en el sector más noroccidental del área de estudio, disipándose hacia el este, aproximadamente a partir del valle del Gállego, a partir del cual predominan las condiciones mediterráneas o mediterráneo-continentales a las que hay que añadir un matiz montano. Tanto las Sierras Interiores como las Exteriores suponen barreras orográficas que originan un descenso muy marcado de las precipitaciones al sur de las mismas. La variedad de influencias y los fuertes gradientes altitudinales dan lugar a importantes diferencias en cuanto a la cantidad de precipitación superando los $2000 \mathrm{~mm}$ anuales en los sectores más elevados mientras apenas se alcanzan los $600 \mathrm{~mm}$ en otros (extremo occidental de la Depresión Interior y cubetas $\mathrm{y}$ fondos de valle de las molasas prepirenaicas).

Las temperaturas están básicamente controladas por el gradiente altitudinal, si bien las zonas con influencia atlántica registran temperaturas medias más bajas. Por encima 
de los $1000 \mathrm{~m}$ las temperaturas medias son inferiores a $10^{\circ} \mathrm{C}$; a $2000 \mathrm{~m}$ la temperatura media se sitúa cerca de los $5^{\circ} \mathrm{C}$ (Puigdefábregas, 1976), y en el periodo de diciembre a marzo la isoterma $0^{\circ} \mathrm{C}$ se sitúa alrededor de $1600 \mathrm{~m}$. (García-Ruiz et al., 1986).

Los regímenes fluviales están determinados por la distribución anual de las precipitaciones y la cantidad de nieve acumulada en la cuenca de drenaje, pues ejerce un importante papel regulador de la precipitación caída durante invierno y primavera.

La Tabla 1 muestra la distribución estacional de las precipitaciones durante el periodo 1964-1994, observándose la existencia de tres grupos de regímenes de lluvia:

\begin{tabular}{|c|c|c|c|c|c|c|}
\hline Localidad & Cuenca & Otoño & Invierno & Primavera & Verano & Anual \\
\hline Aragüés del Puerto & Aragón & 408,2 & 298,6 & 329,3 & 194,4 & 1230,5 \\
\hline Canfranc & Aragón & 589,8 & 484,4 & 487,8 & 296,1 & 1858,1 \\
\hline Bescós & Aragón & 350,8 & 284,3 & 288,9 & 168,9 & 1092,9 \\
\hline Jaca & Aragón & 248,9 & 209,3 & 251,3 & $\overline{164,3}$ & 873,8 \\
\hline Embún & Aragón & 225,4 & 212,3 & 203,1 & 131,8 & 772,6 \\
\hline Artieda & Aragón & 243 & 190,9 & 198,3 & 133,8 & 766 \\
\hline Yesa & Aragón & 267,87 & 235,6 & 237,5 & 120,8 & 861,77 \\
\hline Bal. Panticosa & Gállego & 499,9 & 418,4 & 398,3 & 260,3 & 1576,9 \\
\hline Sallent G. & Gállego & 400,2 & 313,3 & 323,8 & $\overline{225,3}$ & 1262,6 \\
\hline Biescas & Gállego & 373,8 & 291,2 & 301,2 & 210,6 & 1176,8 \\
\hline Sabiñánigo & Gállego & 255,1 & 189,7 & 225,9 & 160,4 & 831,1 \\
\hline Embalse de La Peña & Gállego & 234,6 & 163,3 & 218,2 & 128,8 & 744,9 \\
\hline Ref. Góriz & Ara & 577,29 & $\underline{257,6}$ & 488,11 & $\overline{326,8}$ & 1649,8 \\
\hline Broto & Ara & 376,1 & 278,6 & 308,3 & $\underline{219,8}$ & 1182,8 \\
\hline Fiscal & Ara & 328 & 265,6 & 287,5 & 182,4 & 1063,5 \\
\hline Boltaña & Cinca & 273,18 & 201,2 & 284,01 & 227,7 & 986,09 \\
\hline Molino de Gistaín & Cinca & 313,3 & $\underline{255,9}$ & 318,9 & 283,7 & 1171,8 \\
\hline El Grado & Cinca & 165,8 & $\overline{130,5}$ & 184,6 & 124,4 & 605,3 \\
\hline Seira & Ésera & 275,7 & 209,8 & 337,7 & 257,3 & 1080,5 \\
\hline Graus & Ésera & 170,9 & 132 & 205,6 & 143,8 & 652,3 \\
\hline Caldas de Bohí & Noguera Ribagorzana & 259,23 & $\underline{218,9}$ & 301,4 & 245,8 & 1025,3 \\
\hline Pont de Suert & Noguera Ribagorzana & 246,47 & 190,4 & 274,8 & 203,5 & 915,17 \\
\hline Vilaller & Noguera Ribagorzana & 254,6 & 186,4 & 282 & 222,9 & 945,9 \\
\hline \multicolumn{7}{|c|}{ Máximo estacional } \\
\hline \multicolumn{7}{|c|}{ Mínimo estacional } \\
\hline
\end{tabular}

Las localidades más occidentales (cuenca del río Aragón) en las que se produce un máximo principal en otoño y un máximo secundario muy igualado en primavera e invierno. Los valores más bajos corresponden de forma clara a los meses de verano. Esta distribución es un reflejo de la influencia atlántica con gran actividad frontal desde otoño hasta finales de primavera.

El correspondiente régimen fluvial (río Esca en Sigüés, 510 m, Fig 2b) se define por un caudal abundante a lo largo de todo el invierno, como consecuencia de la escasa retención nival, y un estiaje muy profundo. En cabecera (río Veral en Zuriza, 1187 m, Fig. 2a) la acumulación de nieve es más evidente, con caudales moderados desde octubre a marzo, mientras abril y mayo muestran caudales elevados debido tanto a la fusión como a las lluvias primaverales.

Al sur y este de los sectores oceánicos (Depresión interior y cabecera de los ríos Gállego y Ara) se registran máximos equinocciales con un claro descenso de la precipi- 
tación en invierno, que acusa un mínimo secundario. Este régimen es una respuesta al debilitamiento de las influencias atlánticas y al reforzamiento del anticiclón invernal. El cambio en la distribución de las precipitaciones respecto al grupo anterior, junto a la existencia de mayores altitudes (lo que permite mayor superficie cubierta de nieve durante un periodo más prolongado), explica la existencia de regímenes fluviales muy diferentes. Así, la figura 2c muestra el régimen fluvial del río Ara en Torla a 1033 m, con un máximo de caudal primaveral más acentuado que en los sectores occidentales. El máximo anual se registra en el mes de junio que supera claramente al mes de mayo. Los meses entre diciembre y abril se encuentran claramente por debajo de la media anual con valores semejantes a los registrados en verano, cuando se da la precipitación más baja. Aguas abajo, el río Ara en Boltaña (680 m, Fig. 2d) presenta los caudales más elevados en los meses de mayo y junio. El estiaje invernal es mucho menos marcado que en Torla, pues se reduce la retención nival en el conjunto de la cuenca, siendo en verano cuando se registran los caudales más bajos (García Ruiz et al., 2001).

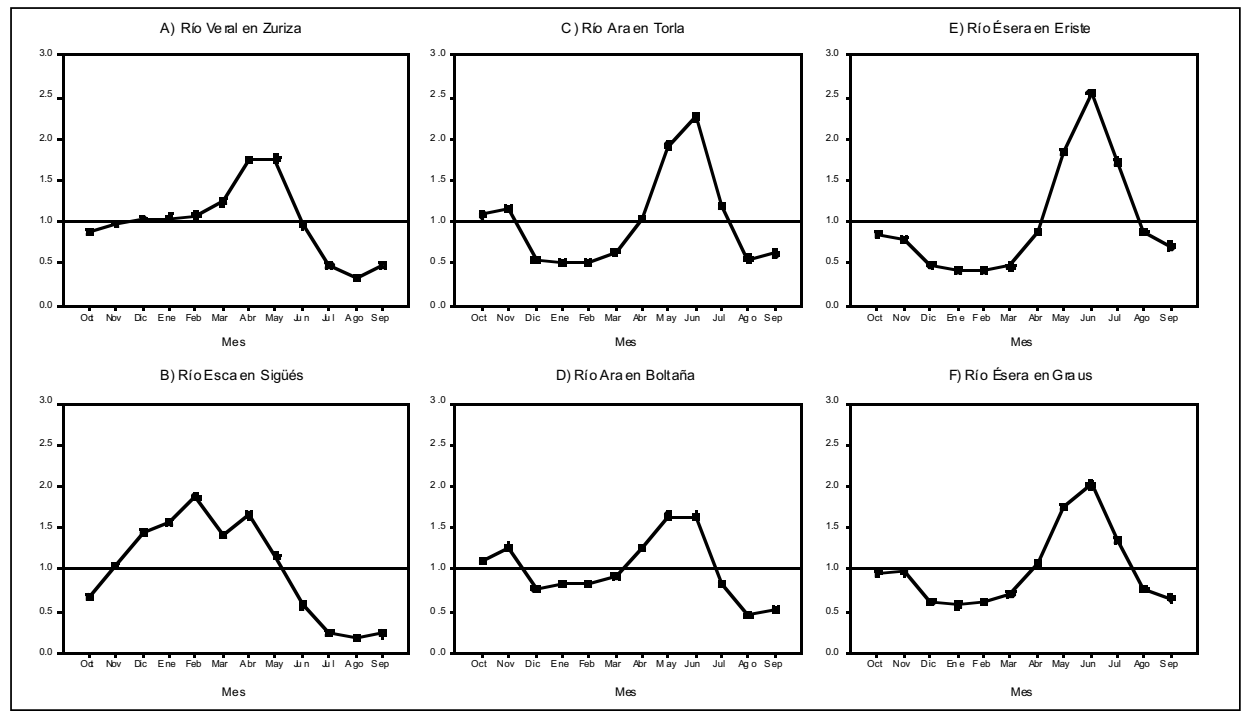

Figura 2. Regímenes fluviales en diversas estaciones de aforo.

iii) Los sectores situados al este del río Ara hasta el Noguera Pallaresa, registran un máximo de precipitación en primavera, seguido de un máximo secundario en otoño. La estación más seca es invierno, lo que introduce un claro matiz continental. En las estaciones de cabecera, la combinación de continentalidad y elevadas altitudes lleva a marcadas puntas de caudal en el mes de junio y un caudal por encima de la media durante el mes de julio. El estiaje invernal presenta caudales más bajos que los registrados en verano, como se observa en la figura 2e (río Ésera en Eriste a $830 \mathrm{~m}$ ). Aguas abajo, en el río Ésera en Graus, a $460 \mathrm{~m}$, el porcentaje de superficie de cuenca ocupada por la nieve se reduce notablemente, por lo que la punta de caudal de junio es menos marcada, con valores semejantes a los de mayo. Los caudales de verano se encuentran ligeramente por debajo de los estivales. Se trata de un régimen similar al de cabecera pero algo suavizado por la influencia submediterránea del curso bajo del Ésera y por una menor influencia de la acumulación de nieve (García Ruiz et al., 2001). 


\section{Material y métodos}

Los datos utilizados han sido facilitados por la Confederación Hidrográfica del Ebro, disponiéndose de valores diarios del volumen almacenado y del caudal a la salida del embalse. Los embalses que cuentan con un canal de derivación disponen de información de las salidas totales del embalse, por un lado, y del caudal derivado hacia el canal, por otro, por lo que el caudal aguas abajo del embalse se ha obtenido restando a las salidas totales el agua derivada hacia el canal.

El caudal de entrada se ha obtenido a partir de la variación diaria del volumen embalsado y las salidas del embalse, de forma que:

$$
\begin{aligned}
& \mathrm{E}_{\mathrm{i}}=\left(\mathrm{V}_{\mathrm{h}}-\mathrm{V}_{\mathrm{i}}\right)+\mathrm{S}_{\mathrm{i}} \\
& \mathrm{Ei}=\text { Aportación al embalse en el día } \mathrm{i} \\
& \mathrm{V}_{\mathrm{i}}=\text { Volumen embalsado en el día } \mathrm{i} \\
& \mathrm{V}_{\mathrm{h}}=\text { Volumen embalsado el día anterior al día } \mathrm{i} \\
& \mathrm{S}_{\mathrm{i}}=\text { Salida del embalse durante día } \mathrm{i}
\end{aligned}
$$

Aunque el procedimiento para obtener la aportación al embalse es sencilla, presenta dos problemas que limitan la fiabilidad de los datos obtenidos:

i) En ocasiones, la estimación del volumen embalsado posee un elevado margen de error, pues se obtiene a partir de la altura de la lámina de agua. Pequeños errores en la medición de su altura suponen importantes errores en la estimación del volumen embalsado. Este problema sucede con mayor frecuencia en aquellos embalses en los que el ratio entre superficie inundada y capacidad de embalsado es bajo, pues la falta de precisión en la estimación de la superficie inundada da lugar a importantes cambios en estimación del volumen existente.

ii) El proceso de aterramiento, que sucede con distinta velocidad en todos los embalses, es otra fuente importante de error en la obtención del volumen almacenado. La relación entre la altura de la lámina de agua y el volumen almacenado se establece a partir de la topografía del vaso del embalse en el momento previo al comienzo de su llenado. Los cambios en la topografía a que da lugar la irregular sedimentación de los sólidos que llegan al embalse, hace variar la relación altura-volumen embalsado, generando problemas en la estimación de las aportaciones al embalse. La solución a este problema está en la realización periódica de batimetrías, que permitan modificar el polinomio que establecería la nueva relación altura de la lámina de agua-volumen embalsado. Si bien el CEDEX (Centro de Estudios y Experimentaciones) lleva a cabo campañas batimétricas anuales, en el Pirineo han sido muy pocas las realizadas, estando la mayor parte de los embalses estudiados sin corregir desde la fecha de su construcción.

Los problemas que surgen en la estimación de la aportación al embalse ha llevado en ocasiones a utilizar como caudales de entrada los valores de caudal registrados en estaciones de aforo cercanas a la cola del embalse, cuando estos existen. Así se ha hecho en 
el embalse de Yesa (suma del río Aragón en la cola del embalse y del río Esca en Sigüés) y en Barasona (suma de los ríos Ésera en Graus e Isábena en Capella).

Al tratarse de infraestructuras gestionadas por el hombre, los datos no se pueden comparar con los de otras series cercanas o regionales, como se hace con los datos climáticos o de aforo. Este hecho dificulta el relleno de datos ausentes y la aceptación o rechazo de datos anómalos, pues en numerosas ocasiones no se puede distinguir si nos encontramos ante un error instrumental o de simple transcripción a formato digital, o son fruto de la actuación de sus gestores (vaciado repentino del embalse, laminación o generación de una crecida, tareas de limpieza en canales o compuertas, etc.).

Una vez realizado un análisis de calidad de los datos, se ha procurado utilizar la mayor cantidad de la información disponible para cada uno de los embalses. En aquellos ríos sobre los que se han instalado más de un embalse, se ha elegido el máximo periodo común a todos los embalses que conforman el sistema. El estudio del cambio del régimen fluvial se ha realizado comparando los regímenes observados aguas arriba y abajo del embalse, frente a la posibilidad de comparar el periodo anterior y posterior a la construcción de la presa en un aforo situado aguas abajo de la misma. Esta última puede introducir errores debido a cambios del régimen fluvial como consecuencia de la variabilidad climática (Krasovskaia y Gottschalk, 2002).

El coeficiente de correlación existente entre los caudales mensuales a la entrada y salida de un embalse o sistema de embalses, ha sido el indicador utilizado para medir la intensidad de cambio de régimen fluvial (Batalla et al., 2002). Un elevado valor de correlación (próximo a 1) indica que el caudal de salida del embalse se encuentra muy condicionado por los caudales de entrada al mismo, por lo que la gestión del embalse apenas supone un cambio en el régimen fluvial. Un coeficiente de correlación bajo (próximo a 0 ) indica que los caudales de salida del embalse tienen muy poca relación con las entradas, indicando que el régimen fluvial experimenta un cambio muy profundo.

\section{Resultados}

\subsection{Características de los embalses pirenaicos}

Han sido estudiados los trece embalses de la zona de estudio que superan los $15 \mathrm{hm}^{3}$ de capacidad. La mayoría de los principales ríos estudiados se encuentran regulados por sistemas múltiples, como los ríos Gállego, Cinca, Noguera Ribagorzana y Noguera Pallaresa. Los ríos Aragón y Ésera están regulados por un embalse principal cercano a las Sierras Exteriores, y pequeños embalses de cabecera destinados a la producción hidroeléctrica. La información de los pequeños embalses de la cabecera del río Gállego se ha agrupado dando lugar a los sistemas Aguas Limpias y Caldarés.

La Tabla 2 muestra las principales características de los embalses estudiados, pudiéndose observar una notable variedad de tipos de embalses en función del uso para el que fueron construidos, capacidad de almacenamiento y ratio de retención. 


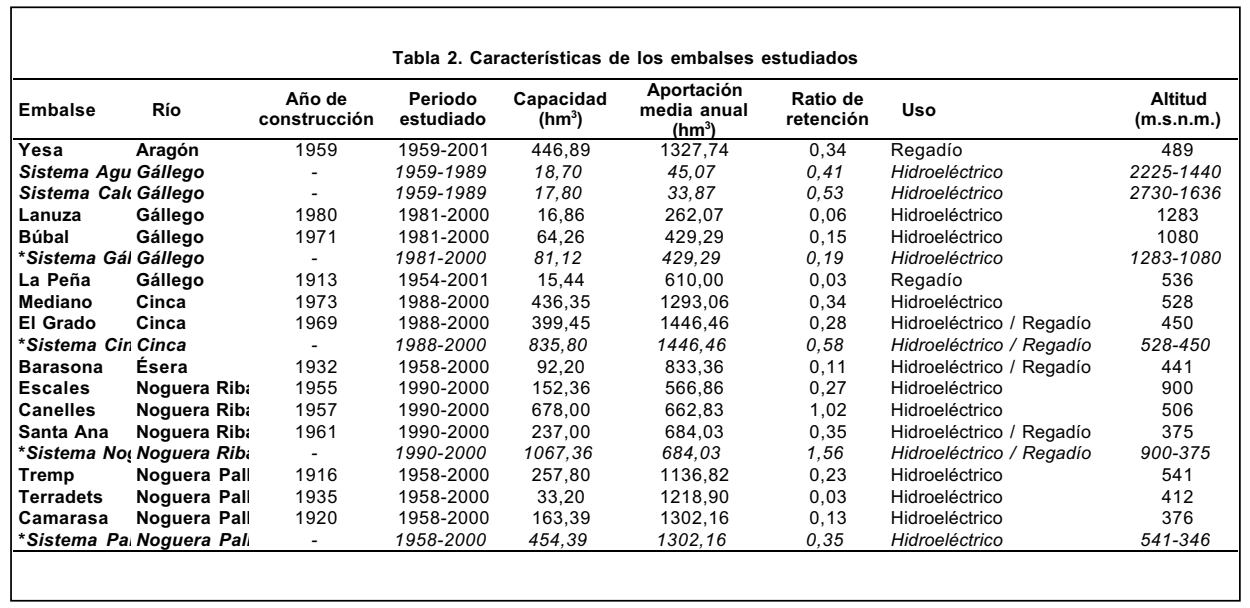

El uso fundamental de los embalses de Yesa y La Peña es el suministro de agua para regadío. Otros como El Grado, Santa Ana y Barasona combinan la producción hidroeléctrica con el suministro de agua para regadío. El resto de los embalses se destinan fundamentalmente a la producción hidroeléctrica.

La capacidad de los embalses estudiados oscila entre 15 y $678 \mathrm{hm}^{3}$. En las zonas de cabecera hasta el sur de las Sierras Interiores, los embalses tienden a ser de reducidas dimensiones y su uso principal es la producción hidroeléctrica. En los grandes afloramientos de litologías blandas, inmediatamente al norte de las Sierras Exteriores, se han instalado los embalses de mayor capacidad, entre los que destacan Canelles $\left(678 \mathrm{hm}^{3}\right)$, Yesa $\left(447 \mathrm{hm}^{3}\right)$ y Mediano $\left(436 \mathrm{hm}^{3}\right)$.

La relación entre el caudal que llega a un embalse o sistema de embalses y su capacidad de embalsado (ratio de retención) es un buen indicador de la capacidad de regulación de cada uno de ellos. El embalse de Canelles es el que muestra un mayor ratio de retención, pues posee mayor capacidad de almacenamiento que el promedio anual de aportación al embalse. Otros embalses como Yesa, Mediano, El Grado, Santa Ana y Escales pueden regular alrededor del 25\% del caudal que reciben. Otros, en cambio, presentan una capacidad muy limitada de regulación, correspondiendo los valores más bajos a embalses de poca capacidad situados en los sectores inferiores de la zona de estudio, como el embalse de La Peña y Terradets. El río Noguera Ribagorzana es el que, con su sistema de tres embalses, presenta mayor ratio de retención (1.56). El río Cinca, Noguera Pallaresa y Aragón muestran ratios intermedios con valores de $0.58,0.35$ y 0.34 respectivamente. El río Gallego posee ratios de retención elevados en sus sistemas de cabecera (Aguas Limpias y Caldarés), pero inmediatamente aguas abajo, los embalses de Lanuza y Búbal presentan una reducida capacidad de almacenamiento en relación al caudal que les llega. Excepto pequeños embalses hidroeléctricos de cabecera, el río Ésera cuenta únicamente con el embalse de Barasona para regular sus aguas. Los $92 \mathrm{hm}^{3}$ de capacidad de este embalse suponen un $11 \%$ del caudal que le llega, siendo el ratio más bajo de la zona de estudio. 


\subsection{Entradas y salidas de agua: Variaciones en el régimen fluvial}

La Tabla 3 muestra los coeficientes de correlación y su significación entre los caudales mensuales registrados a la entrada y la salida de cada uno de los embalses estudiados. Para los embalses que cuentan con canal de derivación se proporcionan dos valores de correlación:

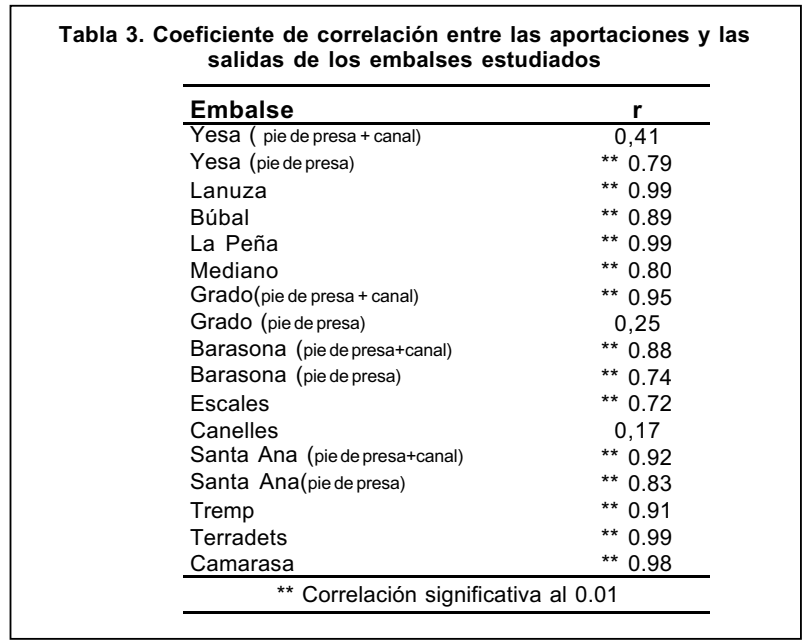

i) entre la aportación al embalse y las salidas totales (agua derivada hacia el canal más el caudal aguas abajo de la presa) que muestra el cambio en la distribución de los caudales entrantes para satisfacer la demanda que se le requiere (irrigación o uso mixto regadío-producción hidroeléctrica).

ii) entre el caudal mensual que entra al embalse y el régimen registrado aguas abajo de la presa, que muestra el cambio de régimen que afecta realmente al curso fluvial.

En la zona de estudio los coeficientes de correlación poseen un rango muy amplio, lo que indica que la intensidad del cambio de régimen fluvial inducido por la acción de los embalses varía notablemente. Varios grupos de embalses pueden distinguirse:

i) En los embalses de Lanuza y La Peña (Fig. 3) la correlación entre el régimen a la entrada y salida del embalse es de 0.99 y estadísticamente significativa al 0.01. En esos casos las salidas del embalse mantienen la misma evolución que las entradas, con pequeñas variaciones que explican las modestas variaciones de volumen embalsado en ambos casos. La reducida capacidad reguladora de ambos embalses permite únicamente mantener los caudales de salida ligeramente más elevados de lo que sería el régimen natural durante los meses de verano. Otros ejemplos de embalses que apenas modifican el régimen fluvial son los de Terradets y Camarasa.

ii) La intensidad de cambio en el régimen hidrológico se hace más patente en otros embalses que presentan un valor algo menor de $\mathrm{r}$ (entre 0.7 y 0.8 ). Así sucede en Tremp, 
Búbal, Barasona, Santa Ana, Yesa y Mediano. A pesar de que la correlación entre el régimen a la entrada y salida de los embalses desciende en algunos casos por debajo de $\mathrm{r}=$ 0.75 , la correlación continúa siendo estadísticamente significativa, indicando que ambos regímenes presentan cierta similitud. La Fig.4 muestra la evolución de los caudales de entrada y salida en los embalses de Mediano (río Cinca) y Escales (río Noguera Ribagorzana), así como el volumen de agua almacenado. Estos dos ejemplos muestran la existencia de periodos en los que las entradas superan a las salidas, impulsando el incremento del volumen embalsado. En el caso del embalse de Mediano se aprovechan las lluvias de otoño, y la fusión nival y lluvias primaverales, mientras que en el embalse de Escales el aumento de volumen se concentra entre abril y julio. El desembalsado de Mediano tiene lugar exclusivamente en verano, para atender a la demanda agrícola de agua. En Escales se producen dos descensos en el almacenamiento, el principal entre enero y marzo y el secundario en verano, en relación con un aprovechamiento hidroeléctrico. Aunque la Fig. 4 muestra la existencia de desfases entre entradas y salidas en ambos embalses, no obstante pueden apreciarse unas tendencias parecidas en los dos parámetros.

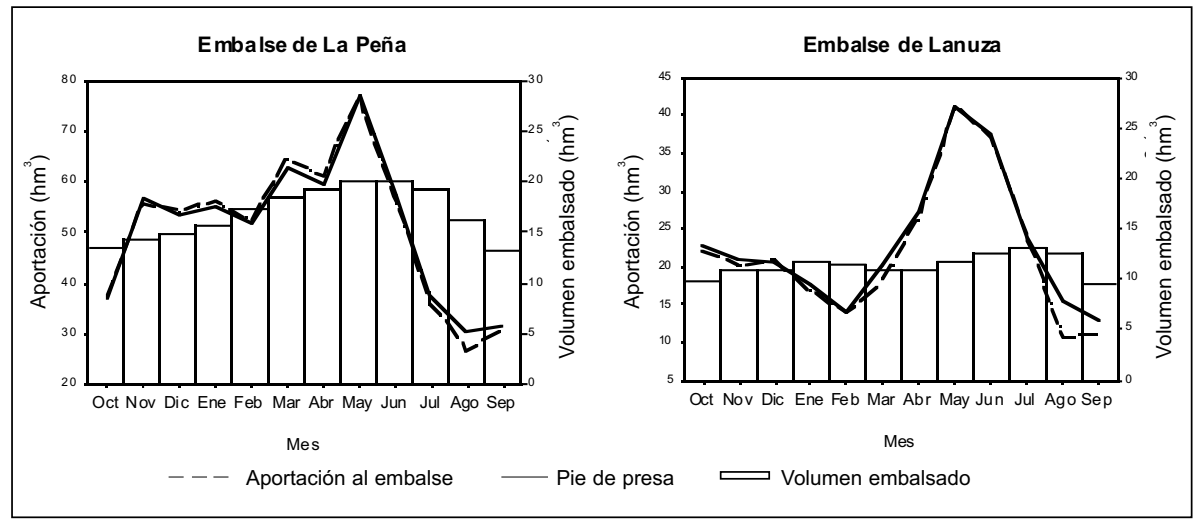

Figura 3. Balance de entradas y salidas de los embalses de La Peña y Lanuza.

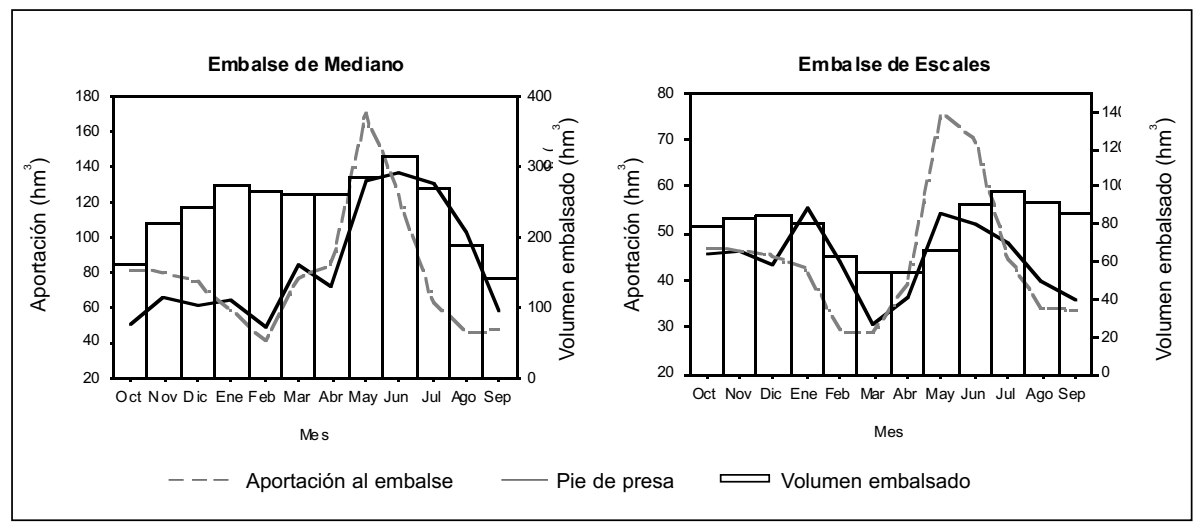

Figura 4. Balance de entradas y salidas de los embalses de Mediano y Escales. 
iii) Un tercer grupo muestra valores de $\mathrm{r}$ inferiores a 0.5 , sin significación estadística. En este grupo se incluyen los embalses que perturban intensamente el régimen fluvial. Así sucede con el embalse de El Grado (Fig. 5) cuando se comparan las entradas, procedentes del embalse de Mediano, y las salidas hacia el río Cinca $(\mathrm{r}=0.25)$. En este caso el régimen de salida amortigua intensamente las grandes diferencias estacionales de caudal que existen en las entradas. De hecho, el gran periodo de aguas altas entre mayo y agosto desaparece por completo y apenas se refleja en un pequeño pico en mayo. De igual forma, el máximo secundario de enero se presenta aguas abajo, de manera muy limitada. Estas diferencias se deben a la derivación de una parte importante del caudal del río Cinca hacia el Canal del Cinca ( $538 \mathrm{hm}^{3}$ anuales), para atender al Plan de Riegos del Alto Aragón (Monegros). En cambio, si se suman las salidas totales (hacia el Canal y hacia el río Cinca), las diferencias entre entradas y salidas son relativamente pequeñas, con especial énfasis en otoño (incremento del almacenamiento de agua embalsada) y en verano (desembalse para riego). De ahí que la correlación entre entradas y salidas totales sea $r=0.95$, es decir un cambio de régimen inferior al que, inmediatamente aguas arriba, produce el embalse de Mediano.

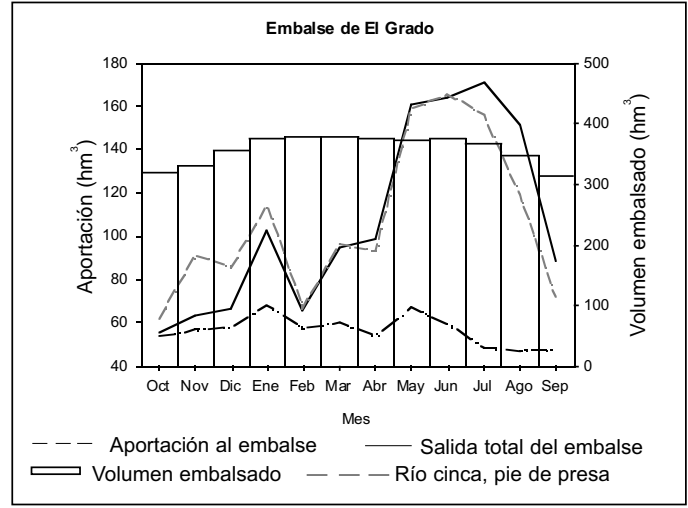

Figura 5. Balance de entradas y salidas del embalse de El Grado.

iv) Por último, el embalse de Canelles (Fig. 6) es el que presenta el coeficiente de correlación más bajo de los embalses estudiados, $(\mathrm{r}=0.17)$. La baja correlación entre la aportación al embalse y el caudal aguas abajo de la presa se basa, en primer lugar, en el almacenamiento de los elevados caudales que se registran durante los meses de otoño, periodo durante el cual se alcanzan los menores caudales aguas abajo de la presa; $y$, en segundo lugar, a las elevadas salidas de caudal entre junio y septiembre, en contraste con las bajas entradas. La Fig. 6 demuestra que en la mayor parte de los meses se producen relaciones inversas entre entradas y salidas, de manera que el río Noguera Ribagorzana aguas abajo del embalse de Canelles puede considerarse completamente alterado por el régimen de almacenamiento y desembalsado.

La Tabla 4 indica el cambio de régimen fluvial que experimenta cada uno de los ríos estudiados debido a la acción de su embalse o sistema de embalses. En este caso la intensidad del cambio de régimen fluvial que genera un sistema de embalses se valora mediante la correlación entre los caudales mensuales que llegan al embalse superior y el régimen registrado aguas abajo del sistema. 
El mayor cambio de régimen fluvial lo producen los sistemas de embalses del Cinca (Embalses de Mediano y El Grado) y Noguera Ribagorzana (Escales, Canelles y Santa Ana) con valores de $\mathrm{r}$ iguales a 0.54 y 0.42 respectivamente. Sistemas de embalses como los instalados en el río Gállego y Noguera Pallaresa dan lugar a cambios poco profundos como lo muestran sus elevados valores de correlación $(\mathrm{r}=0.82$ y $\mathrm{r}=0.88$ respectivamente). Los embalses de Barasona y Yesa, únicos embalses de entidad que regulan los ríos Ésera y Aragón, muestran valores ligeramente inferiores a los anteriormente citados $(\mathrm{r}=0.79$ y 0.74$)$ pero la correlación entre los regímenes fluviales aguas arriba y abajo de los embalses continúa siendo estadísticamente significativa.

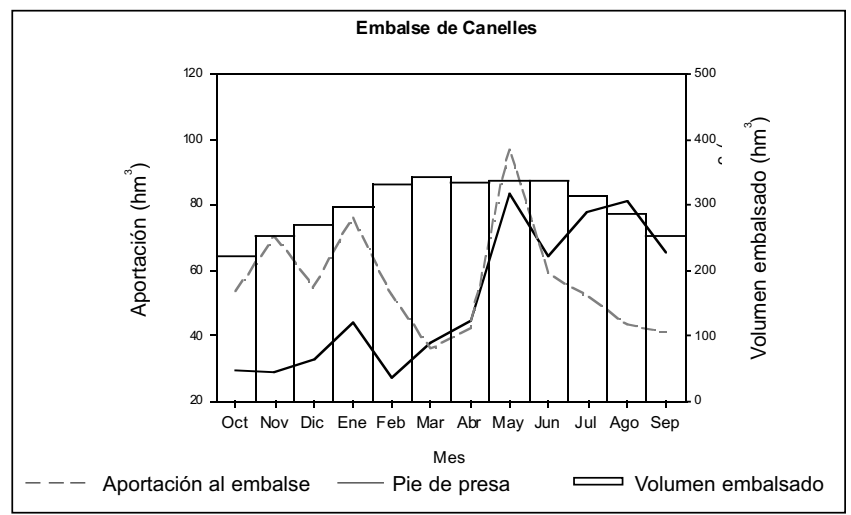

Figura 6. Balance de entradas y salidas del embalse de Canelles.

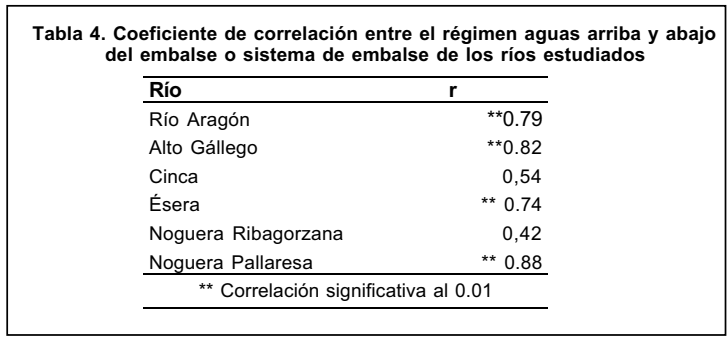

\subsection{Factores condicionantes de las variaciones de régimen fluvial}

La Fig. 7 muestra que la capacidad de embalsado posee elevados valores de correlación con la intensidad de cambio de régimen fluvial. La pendiente negativa de la regresión indica que a mayor capacidad de almacenamiento de agua por parte de un embalse o sistema de embalses, el cambio en su régimen fluvial tiende a ser mayor. La Fig. 8 muestra que el porcentaje de varianza explicada se incrementa de un $41 \%$ a un $69 \%$ cuando la capacidad de embalsado se expresa como un valor relativo, dividiéndolo por la aportación anual que lo alimenta, obteniéndose así su ratio de retención. Un embalse con elevada capacidad de almacenamiento respecto al caudal que recibe posee lógicamente mayor capacidad de cambio de régimen fluvial que otro con un reducido ratio de retención. 
Sin embargo, a pesar del elevado porcentaje de la varianza explicada por el ratio de retención, algunos embalses se comportan de forma diferente a la tendencia general. Un claro ejemplo es la variación del régimen fluvial que genera el embalse de El Grado, pues es mucho mayor que lo que le correspondería en función de su ratio de retención. Este desajuste se debe a que el funcionamiento del embalse de El Grado está muy determinado por la gestión del embalse de Mediano, situado inmediatamente aguas arriba de su cola de embalse. La gestión conjunta de ambos es la que explica la intensidad de cambio del régimen fluvial, así el ratio de retención de este sistema es de 0.58 y la correlación entre entradas y salidas es de $\mathrm{r}=0.54$ por lo que se encontraría dentro de los límites de confianza de la correlación entre ambas variables. La sustitución del valor del embalse de El Grado por el correspondiente al sistema del Cinca hace que el ratio de retención pase a explicar un $84 \%$ de la varianza registrada en la intensidad del cambio de régimen. El resto de los embalses se encuentran dentro o muy cerca de los límites de confianza de la regresión por lo que el ratio de retención se muestra como la principal variable que explica la intensidad con que se produce el cambio de régimen fluvial.

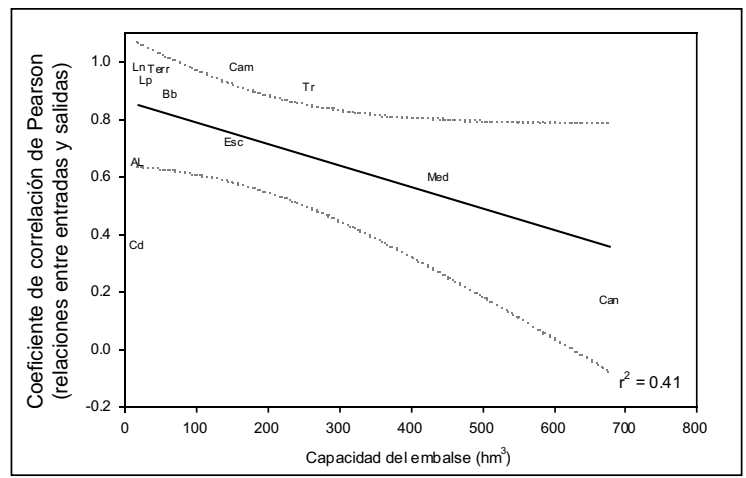

Figura 7. Correlación entre el coeficiente de correlación de Pearson aguas arriba y abajo de los embalses estudiados y su capacidad de almacenamiento de agua. Yes: Yesa, Ln: Lanuza, Lp: Lapeña, Bb: Búbal, Med: Mediano, Gr: Grado, Bar:Barasona, Esc: Escales, Can: Canelles, StA: Santa Ana, Tr. Tremp, Terr: Terradets, Cam: Camarasa, AL: Aguas Limpias, Cd: Caldarés

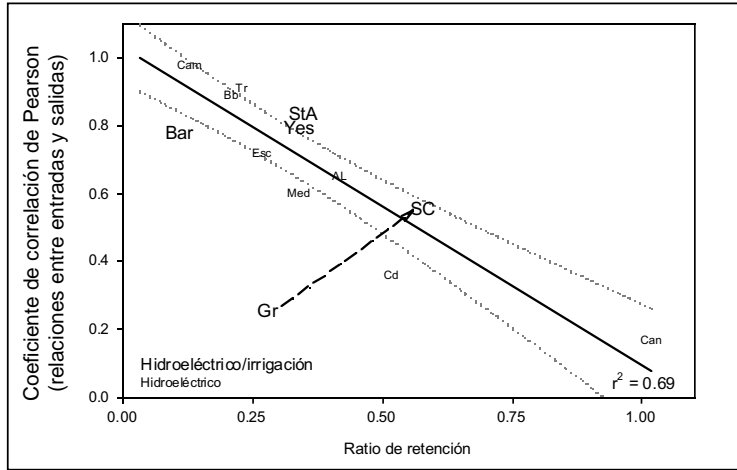

Figura 8. Correlación entre el coeficiente de correlación de Pearson aguas arriba y abajo de los embalses estudiados y su ratio de retención.

Yes: Yesa, Ln: Lanuza, Lp: Lapeña, Bb: Búbal, Med: Mediano, Gr: Grado, SC: Sistema Cinca, Bar:Barasona, Esc: Escales,

Can: Canelles, StA: Santa Ana, Tr: Tremp, Terr: Terradets, Cam: Camarasa, AL: Aguas Limpias, Cd: Caldarés 
La Tabla 5 sugiere que el tipo de actividad a la que se destina el agua almacenada en los embalses contribuye a explicar la distribución estacional de los cambios producidos aguas abajo en el régimen fluvial. En esa Tabla se muestra el signo de la diferencia entre las entradas y las salidas de agua en los embalses. Los meses que no presentan signo indican que el valor de la diferencia se encuentra en torno a 0 (no más de un 5\% del rango de los resultados obtenidos para cada embalse) y por tanto puede decirse que las entradas igualan a las salidas.

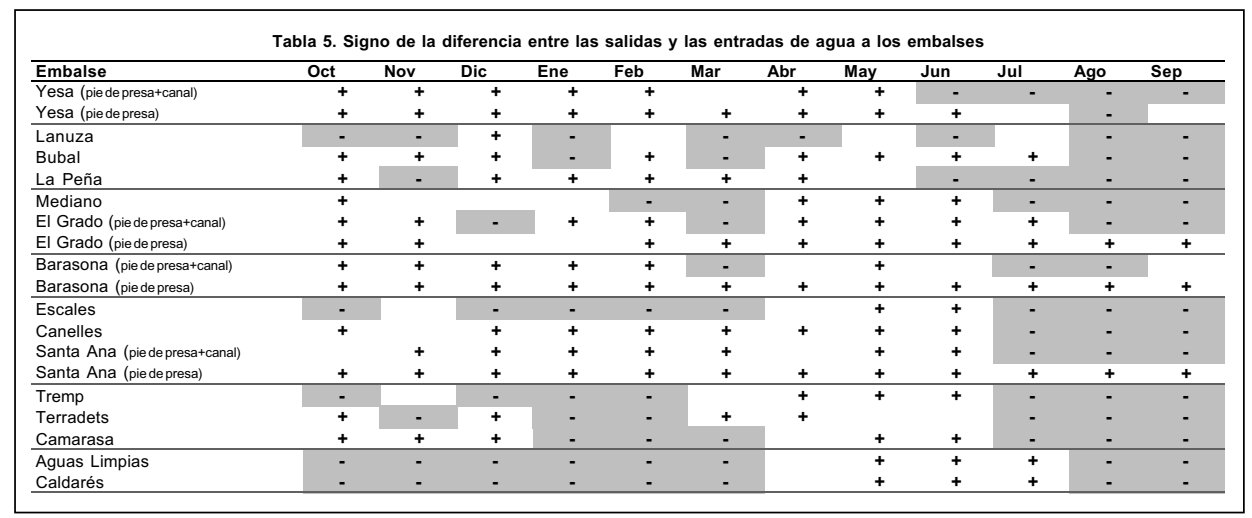

Los embalses destinados únicamente a proporcionar agua para regadío (Yesa y La Peña y Santa Ana en menor medida), almacenan agua durante un largo periodo, entre octubre y mayo, aprovechando los caudales elevados de otoño y primavera e incluso los de invierno, debido a la relativa influencia oceánica. De esta manera disponen del máximo volumen de agua almacenada a finales de la estación húmeda y comienzos de la seca, cuando tienen que proporcionar los mayores caudales posibles a las áreas de regadío (entre junio y septiembre). En cierto modo puede afirmarse que los embalses para riego responden a un modelo muy simple, con un solo periodo de almacenamiento y otro, más corto, de desembalsado, coincidiendo respectivamente con la estación húmeda y la seca.

El comportamiento de los embalses hidroeléctricos es más complejo, puesto que además de adaptarse a las condiciones hidrológicas tienen que hacerlo también a la demanda de electricidad. Así los embalses de Lanuza y Búbal (en la cabecera del río Gállego) muestran frecuentemente volúmenes de salida más elevados que los de entrada desde octubre hasta abril con el fin de atender la demanda energética invernal. También, como es lógico, presentan más salidas que entradas en agosto y septiembre, debido al descenso natural de las aportaciones de la cuenca. Lo mismo sucede, con algunas variaciones, con los embalses de Mediano (río Cinca) y Escales (río Noguera Ribagorzana) y Tremp, Terradets y Camarasa (río Noguera Pallaresa), con importantes salidas de caudal en pleno invierno, y almacenamiento durante un corto, pero muy acusado, periodo primaveral de aguas altas. Los embalses de Aguas Limpias y Caldarés, en el Alto Gállego, funcionan de forma muy similar, con periodo de llenado entre mayo y junio y desembalsado a partir de agosto hasta el mes de marzo.

En el embalse de Barasona (río Ésera), con aprovechamiento mixto regadío/ hidroeléctrico y con dimensiones relativamente reducidas, se optimiza la producción hidroeléctrica a lo largo de todo el año, pero aumenta el desembalsado en marzo con recuperación en primavera y agotamiento de reservas en verano. 


\section{Conclusiones}

Los embalses producen importantes cambios en el régimen fluvial debido a una gestión que tiende a aprovechar, en general, los periodos de caudales más elevados para almacenar agua, mientras los momentos de caudal más bajos se definen por el predominio de los desembalses. Sin embargo cuando se analizan las cuencas y los embalses de manera más individualizada, el ritmo de embalsado y desembalsado (así como su influencia en el régimen fluvial) se encuentra muy influido por dos factores: i) las dimensiones del embalse, sobre todo en relación con las aportaciones o ratio de retención y, ii) el uso prioritario al que se destina el agua almacenada.

El ratio de retención es un buen indicador de la capacidad de regulación que posee un embalse y explica gran parte de la varianza que muestra la intensidad del cambio hidrológico observado en los ríos estudiados. Este trabajo ha demostrado la existencia de una gran variabilidad en el efecto hidrológico de los embalses, desde aquellos que apenas introducen cambios (La Peña, Lanuza, Búbal) hasta los que alteran casi completamente el régimen fluvial (Canelles). Indudablemente, los sistemas complejos con varios embalses son los que introducen una mayor perturbación (sistema Mediano-El Grado, en el río Cinca, y sistema del río Noguera Ribagorzana, especialmente).

Por su parte, el uso prioritario de cada embalse contribuye a explicar los tipos de cambios que se producen a lo largo del año. El modelo más simple corresponde a los embalses para regadío, con llenado entre octubre y mayo y desembalse estival. Los embalses de uso hidroeléctrico o mixto muestran comportamientos más diversificados, que tienen en cuenta tanto el régimen de entradas al embalse como la demanda de energía eléctrica, e incluso en algunos casos, la demanda agrícola.

\section{Agradecimientos}

Este trabajo se ha realizado con el apoyo de los siguientes proyectos de investigación: "Water resources management in a changing environment; The impact of sediment on sustainability"-WARMICE (ENV-CT98-0789), "Debrisfall assesment in mountain catchments for local end users"- DAMOCLES (EVG1-1999-00027P), financiados por la Comisión Europea, y "Efectos hidrológicos de los cambios de uso del suelo a diferentes escalas espaciales" - HIDROESCALA (REN2000-1709-C04-01/GLO) y "Procesos hidrológicos en áreas seminaturales mediterráneas"-PROHISEM (REN2001-2268-CO201/HID), financiados por la CICYT.

\section{Bibliografía}

ADAms, W.M. (1985). The downstream impacts of dam construction: a case study from Nigeria. Trans. Inst. Br. Geogr., 10: 292-302

Al-TAiEe, T.M. (1990). The influence of a dam on the downstream degradation of a river bed: case study of the Tigris River. In: Hydrology in Mountainous Regions IIArtificial Reservoirs; Water and Slopes, IAHS Publ.,194: 153-160.

ANDrEws, E.D. (1986). Downstream effects of Flaming Gorge reservoir on the Green River, Colorado and Utah. Geological Survey of America Bulletin, 97: 1012-1023. 
Batalla, R.J., Kondolf, G.M. y Gómez, C.M. (2002). Hydrological alterations in the Ebro Basin caused by dams. Journal of Hydrology (en revisión)

BAXTER, R.M. (1977). Environmental effects of dams and impoundments. Ann. Rev. Ecol. Syst, 8: 255-283.

BonacCI, O. y RoJE-BonacCI, T. (2003). The influence of hydroelectrical development on the flow regime of the Karstic river Cetina. Hydrological Processes, 17 (1): 1-15

BRANDT, S. (2000). Classification of geomorphological effects downstream of dams. Catena, 40: 375-401.

García-Ruiz, J.M., Puigdefabregas, J. y Creus, J. (1986). La acumulación de nieve en el Pirineo Central y su influencia hidrológica. Pirineos, 127, 27-72.

García-Ruiz, J.M, Beguería, S., López-Moreno, J.I., Lorente, A. Y Seeger, M. (2001). Los recursos hídricos superficiales del Pirineo aragonés y su evolución reciente. Ediciones Geoforma, 192 p, Logroño.

Giers, A., Freistüler, E. y Schultz, G. (1998). Methodology for assesment of ecohidrological effects of dam construction in a headwater region. Hydrology, Water Resources and Ecology in Headwaters, IAHS Publ., 248: 509-514.

Higgs, G. Y Petts, G. (1988). Hydrological changes and river regulation in the UK. Regulated Rivers: Rearch and Management, 2: 349-368.

KrasovsKaiA, I. y GotTSCHALK, L. (2002). River flow regimes in a changing climate. Hydrological Sciences Journal, 47 (4): 597-609

MorRIS, G.L. y FAN, J. (1997). Reservoir Sedimentation Handbook. McGraw-Hill.

López Moreno, J.I., Beguería, S.y García Ruiz, J.M. (2002). Influence of the Yesa reservoir on floods of the Aragón River, central Spanish Pyrenees. Hydrology and Earth System Sciences, 6 (4): 753-762.

PIRCHER, W.(1990). The contribution of hydropower reservoirs to flood control in the Austrian Alps. In: Hydrology in Mountainous Regions. II Artificial Reservoirs: Water and Slopes., IAHS Publ., 194: 3-10.

Puigdefábregas, J. (1976). Pautas espaciales de variación climática en el Alto Aragón. Publ.Centro Pir. Biol. Exp, 7 (1): 23-34.

SAMmUt, J. Y ERSKINE, D. (1995). Hydrological impacts of flow regulation associated with the Upper Nepean water scheme, NSW. Australian Geographer, 26 (1): 71-86.

TAKEUCHI, K., (1998). Current status of reservoirs: Number, size and shape of reservoirs. In K.Takeuchi, M. Hamlin, Z.W. Kundzewicz, D Rosbjerg \& S.P. Simonovic (eds.), Sustainable reservoir development and management, IAHS Publ., 251: 4-7.

ZSUFFA, I. (1999). Impact of Austrian hydropower plants on the flood control safety of the Hungarian Danube reach. Hydrological Sciences Journal, 44(3): 363-371. 